\title{
Comparación de dos metodologías de enseñanza-aprendizaje en waterpolo Comparison of two teaching-learning methodologies in water polo
}

*Pablo José Borges Hernández, **Encarnación Ruiz Lara, *Francisco Manuel Argudo-Iturriaga

*Universidad Autónoma de Madrid (España), ** Universidad Católica de Murcia (España)

Resumen. El objetivo del estudio fue analizar si el uso de las TIC, asociado a una metodología de enseñanza comprensiva, influye en el aprendizaje del waterpolo en categoría benjamín y alevín. Se diseñaron y aplicaron 10 sesiones de 30 minutos sobre pase, recepción y desmarque. Un grupo de 6 jugadores, de cada categoría, participó en un proceso de enseñanza-aprendizaje utilizando dos aplicaciones informáticas, mientras que otro grupo, del mismo número de jugadores y categoría, lo hizo exclusivamente con indicaciones orales del entrenador. Se realizó un pre-test de 20 situaciones del juego de los 10 pases, un proceso de enseñanza-aprendizaje y un post-test idéntico. Se empleó la prueba U de Mann-Whittney de comparación entre grupos (pre-post y TIC vs. comunicación oral) en relación al número de pases, duración de la jugada o posesiones, número de pérdidas y recuperaciones, y resultado final del juego. Hallando diferencias significativas entre los diferentes grupos y ambos test en las dos categorías $(p=.000 p<.001)$. Al considerar los análisis en función de la intervención realizada en ambas categorías se aprecian diferencias para el grupo con uso de las TIC entre el número de pases, con y sin posesión de balón $(p=.017 p<.05)$; mientras que en el grupo con comunicación oral se hallan diferencias en el tiempo, tanto con posesión como sin posesión de balón $(p=.001)$. Se concluye que el uso de las TIC ofrece mejores resultados en el aprendizaje de algunas acciones técnicas y su aplicación en la dinámica del juego que la comunicación oral.

Palabras clave: Enseñanza, Iniciación deportiva, Software, Técnica, Waterpolo

Abstract. The aim of this study was to analyze if the use of ICT, along with a comprehensive teaching methodology, influences the learning of water polo in U10 and U12 category. 10 sessions of 30 minutes each, focusing on pass, reception, and getting away from markers, were designed and applied. A group of 6 players from each category participated in a process of teaching-learning based on two software programs, whereas another similar group received oral coach indications exclusively. The pre-test consisted of 20 situations of the 10-pass game, followed by the teaching-learning period and finally by a post-test, identical to the pre-test. Subsequently, MannWhittney U test was used for comparison between groups (pre-post and ICT vs. oral communication) in relation to the number and time of possessions, losses and recoveries, and final result of the game. Significant differences were found between the groups and both tests in two categories $(p=.000 p<.001)$. The analysis based on the intervention program applied in both categories showed significant differences for the ICT group in the number of passes, with and without ball possession $(p=.017 p<.05)$; while for the oral communication group differences were found in time, both with and without ball possession $(p=.001)$. In conclusion, the use of ICT

\section{Introducción}

El cambio en las metodologías de enseñanza-aprendizaje evolucionan a la par que el desarrollo de Internet y las herramientas Web (Cabero \& Marín, 2013). Por lo que está bastante asentado reconocer los valores positivos y la actitud favorable de los jóvenes hacia las TIC’s (Guillen, Herrera \& de la Rosa, 2018), las redes sociales (Gómez, Roses \& Farias, 2012) y las nuevas tecnologías en su formación(Tejedor, García-Valcárcel \& Prada, 2009), los cual no queda al margen del desarrollo deportivo y la contemporaneidad. Por ello, esta propuesta intenta medir como afecta el uso de las TIC y diversas metodologías de enseñanza en el aprendizaje de diferentes contenidos técnico-tácticos en el contexto deportivo.

Y es que en la actualidad esto elementos se han convertido en un fuerte aliado para unir lo que los jóvenes consideran entretenimiento, con la formación y el aprendizaje (Colás, González \& de Pablo, 2013). Por lo que este trabajo pretende unir el aprendizaje de habilidades deportivas haciendo uso de nuevas tecnologías y las redes sociales y fomentar el aprendizaje colaborativo de los discentes, en una sociedad cada vez más conectada, en la cual aprender a trabajar con otros y colaborar se convierte en una competencia trascendental (GarcíaValcárcel, Basilotta \& López, 2014; Marín, Negre \& Pérez, 2013). Y que acentúa la interacción didáctica y formativa, la atención individualizada, y la retroalimentación de la dinámica deportiva de forma coherente y objetiva (Guillen, Herrera \& de la Rosa, 2018).

Específicamente en el ámbito deportivo, la preocupación por su enseñanza, especialmente en sus etapas de iniciación, se compromete muy de cerca el conocimiento de lo novedoso y lo contemporáneo que se deriva del contexto pedagógico y las formas de gestionarlos (Guillen, Copello, Gutierrez \& Guerra, 2018). Ocupando desde hace tiempo a

Fecha recepción: 30-06-18. Fecha de aceptación: 22-11-18 Pablo José Borges Hernández pablo.borges@externo.uam.es maestros, entrenadores e investigadores delámbito deportivo(Carmona, Luján, Francisco \& Olmedilla, 2015; López \& Castejón, 2005, Meroño, Calderón \& Hastie, 2016), quienes reclaman una continua actualización en el orden teórico, metodológico y práctico (Guillen et al., 2018). Tradicionalmente se han esbozado dos corrientes antagónicas: una tradicional, basada en la técnica y que incide en mayor medida en un domino técnico básico, y que se fundamenta en el uso de la instrucción directa por parte del profesor exigiendo una escasa implicación en el desarrollo cognitivo y motriz del alumno (González, Ibañez, Feu \& Galatti, 2017); y que va del cómo al qué(el cómo sería la técnica y el qué la táctica), o bien con estrategias globales, más tácticas (del quéal cómo), que se basan en la lógica o estructura del deporte (Castejón, 2015), se basan en la implicación cognitiva del alumno, mediante el empleo del descubrimiento y la resolución de problemas (Abad, Benito, Giménez \& Robles, 2013) y que surgen al albor de las nuevas propuestas educativas producto de la revolución de la pedagogía constructivista (Piaget, 1982) y la enseñanza comprensiva del deporte (Blomqvist, Luhtanen \& Laakso, 2001; Serra \& García, 2017).

Producto de esta nueva concepción de la educación deportiva y en la búsqueda de la resolución de la insatisfacción docente que suscitaba la enseñanza deportiva centrada en los gestos técnicos (Bunker \& Thorpe, 1982) y las sesiones repetitivas y aburridas que alejaban al alumnado de los juegos deportivos, especialmente a las chicas (Sevil, Abós, Julián, Murillo \& García, 2015) y a las personas menos hábiles, han aparecido diferentes corrientes y modelos de enseñanza, destacando el propuesto por Bayer (1986) y la praxiología motriz de Parlebàs (1981) desde el ámbito francés. A su vez, han surgido las aportaciones del modelo de enseñanza comprensiva (Teaching Games for Understanding, TGfU) desde el Reino Unido (Bunker \& Thorpe, 1982; Thorpe, Bunker \& Almond, 1986) y sus variantes: Game Sense y Play Practice desde Australia (Light \& Robert, 2010; Light \& Tan, 2006), Games Concept Approach desde Singapur (Rossi, Fry, McNeill \& Tan, 2007), Tactical Games Approach desde EE.UU (Gubacs-Collins, 2007) y Tactical Decision Learning Model o Invasion Games Competence Model (Tallir, Lenoir, Valcke \& Musch, 2007) también desde Francia, así como han aparecido diferentes propuestas en España (Abad et al., 2013; Castejón, 
Martínez, del Campo \& Argudo, 2011) cuyo objetivo ha sido describir y analizar los fundamentos teóricos más relevantes sobre los que se apoya la enseñanza comprensiva del deporte atendiendo a la literatura científica existente. Por este motivo, documentan 54 referencias relativas a la enseñanza del deporte de forma comprensiva (Abad et al., 2013) y cuyo fin es desarrollar la comprensión del juego enfatizando las interacciones del aprendiz dentro del entorno en la construcción de conocimiento, a través de la conciencia táctica y de la apreciación del juego (Gray \& Sproule, 2011), lo cual ayudará a los alumnos/jugadores a ser competentes en el deporte (Holt, Ward \& Wallhead, 2006).

A raíz de estas aportaciones y tal y como se entiende la educación y formación deportiva actual, el proceso de enseñanza-aprendizaje y/o entrenamiento deportivo debe organizarse a partir del conocimiento de la naturaleza del juego. Ello permitiráuna correspondencia entre la lógica interna y la lógica didáctica que promueva actividades de enseñanzaaprendizaje que contengan esta naturaleza y favorezcan la transferencia de lo aprendido a la competición (Jiménez, 2011). Donde destaca el papel de la atención, la memoria, la planificación, la retroalimentación en las acciones motrices y deportivas; y la necesidad de dotar de una participación activa al aprendiz/deportista puesto que no se busca la repetición mecánica de un determinado movimiento, sino que se busca desarrollar un aprendizaje que le permita solucionar los problemas motores y situaciones que se suceden en la competición por él mismo (del Valle, Mendoza, Sánchez \& de la Vega, 2007). Recientemente, el estudio de los condicionantes de las tareas respecto a su esencia y repercusión en competición ha permitido demostrar que la dinámica del juego está más influenciada por los problemas tácticos del deporte, que por otros condicionantes como el espacio o el número de jugadores (Serra \& García, 2017).

Lamentablemente, una revisión de la literatura actual destaca la falta de trabajos empíricos para comprobar la eficacia de los programas planteados en la iniciación deportiva (Abad et al., 2013; Febré, Chirosa, Casamichana, Chirosa, Marín \& Pablos, 2015), aunque ha sido uno de los ámbitos más estudiados (González et al., 2017). En estos trabajos, se observa la falta de preparación de los técnicos que se encargan de la iniciación deportiva en edades tempranas (Castejón et al., 2011). A su vez, Gordillo (1992) expone que, debido al desconocimiento y falta de adecuación y contextualización de las exigencias del entrenador a sus deportistas, en muchas ocasiones muy alejadas de la evolución y maduración física y psicológica del deportista, desemboca en falta de motivación y abandono de los mismos. Todo ello, motiva que Sevil et al.(2015) apunten que es necesaria la realización de programas de intervención que valoren el aprendizaje y los efectos psicológicos del entrenamiento.

Las propuestas de iniciación deportiva al waterpolo siempre han seguido una dinámica descriptiva (García, 2009) no conociéndose ningún estudio experimental o cuasi-experimental que se haya interesado por conocer cómo influye la estrategia de enseñanza en el aprendizaje y mejora de la formación deportiva en esta modalidad acuática. Si alzamos la mirada al resto de deportes observamos que durante los últimos años ha surgido, por parte de maestros, entrenadores e investigadores del ámbito deportivo, una interesante preocupación por su enseñanza, especialmente en sus etapas de iniciación (López \& Castejón, 2005). En este sentido se tiene conocimiento de dos aproximaciones o corrientes antagónicas de la enseñanza deportiva. Una tradicional basada en la técnica y otra proveniente de la pedagogía constructivista (Piaget, 1982) basada en el predominio de la enseñanza de la táctica (Blomqvist, Luhtanen \& Laakso, 2001) y la comprensión del juego. De ahíla importancia dentro de la planificación deportiva de la inclusión y uso de juegos que simulen total o parcialmente la competición. Lo cual se considera como una estrategia de gran valor en la mejora del rendimiento (Katis \& Kellis, 2009) y reciben el nombre de small-sided games (Rampinini et al., 2007).

Por este motivo, y buscando la mejora de los procesos de enseñanza-aprendizaje deportivos, el objetivo de este estudio fue analizar si el uso una metodología de enseñanza-aprendizaje comprensiva, asociada al uso de las nuevas tecnologías y el empleo de grupos de discusión para el aprendizaje de la técnica y a táctica deportiva, influye en el aprendizaje de la dinámica del juego en waterpolo, en las categorías benjamín y alevín. Frente a una vía oral de comunicación y el empleo de una metodología tradicional exclusivamente, basada en la enseñanza de la técnica, el control de balón y la técnica de nado.

\section{Metodología}

\section{Participantes}

La muestra estuvo compuesta por 12 jugadores de categoría benjamín (9-10 años) y otros tantos de categoría alevín (11-12 años) con dos y cuatro años de experiencia en la práctica del waterpolo respectivamente, seleccionados de forma incidental bajo el único criterio de fácil acceso. Se trataba de unos grupos homogéneos desde el punto de vista físico, técnico y táctico y en fase de aprendizaje del deporte; motivo por el cual fueron seleccionados para este estudio, en el que participaron de forma voluntaria. Posteriormente, en cada categoría, fueron distribuidos en dos grupos de forma aleatoria, de cara a la participación en la intervención con una u otra vía de comunicación.

Con respecto a las características del entrenador, que participó de manera voluntaria y altruista en este estudio, tiene formación universitaria en esta modalidad deportiva acuática y posee el título deportivo de máximo nivel que expide la Real Federación Española de Natación. Además, tiene una trayectoria como técnico de más de diez años en la que ha cosechado grandes resultados a nivel nacional en estas categorías.

\section{Diseño}

Se utilizó la metodología observacional (Anguera, 2003) mediante un diseño descriptivo y correlacional de carácter transversal cuasi-experimental pre-post, dos grupos (Montero \& León, 2007), para determinar si existen diferencias entre un proceso de enseñanza-aprendizaje deportivo basado en uso de las nuevas tecnologías (NT) y otro basado en la comunicación oral (CO), según la clasificación recogida en Abad (2008) y Robles (2006).

Por estos motivos, en este estudio se analizaron cuatro variables y sus núcleos categoriales:

1-Número de pases, tanto en posesión de balón como sin posesión de balón (ésta última se refiere a los pases que hace el grupo contrario).

2-Número de balones perdidos y recuperaciones efectuadas.

3-Duración de la jugada (media \pm desviación típica, expresada en segundos), tanto en el caso de las situaciones donde se posee balón, como en las situaciones en que no se posee.

4-Resultado final de cada situación de juego: ganador o perdedor (referido al equipo que logra conectar 10 pases).

\section{Procedimiento}

Se realizó un pre-test compuesto por 20 situaciones de juego de los «10 pases» en el medio acuático, 10 con inicio de posesión para cada grupo. Posteriormente cada uno de los grupos participó en 10 sesiones de aprendizaje específico del waterpolo de 30 minutos de duración, cuyos contenidos giraron en torno a las diferentes modalidades técnicas de ejecución de tres acciones motrices (pase, recepción y desmarque). El primer grupo trabajó empleando un canal de comunicación visual usando las TIC. Mientras, el segundo grupo lo hizo mediante un canal de comunicación oral. En ambos casos las diversas acciones motrices se desarrollaron por parejas, con y/o sin balón y sin oposición; aportando el entrenador un feedback individual y prescriptivo. Los campos de juego en los que se desarrollaron las acciones consideradas tenían unas dimensiones de 15x10 m en la categoría benjamín, mientras que fue de 20x12.50 m en la categoría alevín. Finalmente se realizó un post-test idéntico al pre-test. 
Intervención pedagógica.

En relación a la intervención pedagógica desarrollada, las sesiones de aprendizaje para uno de los grupos consistieron en la observación de las imágenes de las acciones a realizar, por parte de los jugadores, las respuestas del entrenador si había alguna duda al respecto y la realización de las tareas propuestas. Cabe decir que los modelos que observaban eran integrantes del equipo nacional. Mientras que, para el otro grupo, el entrenador describía las acciones y tareas a realizar de forma oral, respondía a las posibles cuestiones planteadas por los jugadores y la ejecución de las mismas. Si durante la realización de las tareas el entrenador identificaba algún aspecto técnico que se estuviera llevando a cabo de forma incorrecta, su intervención con el primer grupo consistía en enseñar las imágenes de la aplicación informática. Mientras que si se trataba de algún integrante del segundo grupo su actuación era de forma oral.

\section{Material}

Los balones utilizados fueron los reglamentarios de ambas categorías: Talla 2 o minisize en la primera y Talla 3 o Junior en la segunda, ambos de la marca Turbo.

Se grabaron y analizaron las 40 situaciones del juego de los «10 pases» de cada categoría. La filmación fue realizada con una cámara de vídeo digital (JVC, GZ-MG50E, JAPAN), ubicada en una posición elevada sobre el espacio de juego, para su posterior análisis por parte de varios observadores no vinculados al estudio con más de 500 horas de experiencia y previamente entrenados. La fiabilidad entre observadores fue verificada usando el índice de kappa (León \& Montero, 2003), alcanzando un valor superior a .85.

Para la enseñanza de las acciones motrices se utilizó una aplicación informática que mostraba una secuencia de imágenes, tanto aéreas como submarinas, de cada una de las mismas (todos los modelos eran jugadores del equipo nacional masculino absoluto). Mientras que todas las situaciones de juego fueron analizadas mediante el software Polo partido v1.0 (Argudo, Alonso \& Fuentes, 2005).

\section{Análisis estadístico}

Se emplearon tablas de contingencia para el estudio de los resultados en relación al número de posesiones, el número de pases en posesión del balón y sin posesión de balón, el número de pérdidas y recuperaciones y la duración de la jugada, empleándose la prueba U de MannWhittney para comparación entre grupos (pre-test - post-test, grupo NT - grupo CO) como estadístico. Los tamaños del efecto (TE) fueron calculados con el estadístico D de Cohen y su interpretación se basó en el siguiente criterio: .01 efecto pequeño, .06 efecto medio, y .14 efecto grande (Cohen, 1988). El nivel de significación fue fijado en $p<.05$, empleando el paquete estadístico SPSS versión 21.0 (IBM Corp., Armonk, NY, USA) para el análisis.

\section{Resultados}

La Tabla 1 muestra los resultados referentes al número de posesiones, los pases realizados por los grupos cuando están en posesión del balón y cuando no, las pérdidas y recuperaciones, la duración media de las situaciones tanto en cuanto se actuaba como grupo poseedor del balón, como cuando se trataba de recuperarlo, en función de la intervención realizada (NT o CO) y el momento de análisis (pre-test o post-test) en función de la categoría de los participantes (benjamin o alevin).
Tabla 1.

te las situaciones acaecidas en el juego de los 10 pases categoria

Benjan $\quad$ NT Pre-test Post-test \begin{tabular}{ccccccccccc} 
& M & DT & M & DT & M & DT & M & DT & M & DT \\
\hline Posesiones & 9,05 & 3,15 & 9,13 & 2,81 & 8,98 & 3,24 & 10,05 & 3,30 & 8,05 & 2,68
\end{tabular} $\begin{array}{lllllllllll}\text { Pases Posesión } & 38,96 & 18,68 & 47,08 & 17,08 & 30,85 & 16,84 & 40,95 & 19,84 & 36,98 & 17,46\end{array}$ $\begin{array}{lllllllllll}\text { Pases Sin Posesión } & 38,96 & 18,68 & 30,85 & 16,84 & 47,08 & 17,08 & 40,95 & 19,84 & 36,98 & 17,46\end{array}$ $\begin{array}{lllllllllll}\text { Perdidas } & 8,55 & 3,15 & 8,48 & 3,13 & 8,63 & 2,91 & 9,55 & 3,30 & 7,55 & 2,67\end{array}$ $\begin{array}{lllllllllll}\text { Recuperaciones } & 8,55 & 3,15 & 8,63 & 2,91 & 8,48 & 3,13 & 9,55 & 3,30 & 7,55 & 2,67\end{array}$ Tiempo $\quad 248,43 \quad 65,53 \quad 248,43 \quad 66,24 \quad 248,43 \quad 66,24 \quad 256,85$ 65,90 240,00 64,88

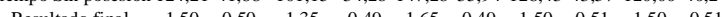

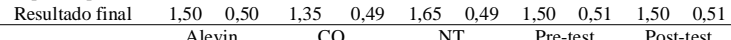
\begin{tabular}{cccccccccc}
\multicolumn{1}{c}{ Alevin } & \multicolumn{2}{c}{ CO } & \multicolumn{3}{c}{ NT } & \multicolumn{2}{c}{ Pre-test } & \multicolumn{2}{c}{ Post-test } \\
M & DT & M & DT & M & DT & M & DT & M & DT \\
\hline
\end{tabular}

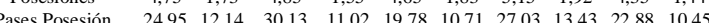
$\begin{array}{lllllllllll}\text { Pases Posesión } & 24,95 & 12,14 & 30,13 & 11,02 & 19,78 & 10,71 & 27,03 & 13,43 & 22,88 & 10,45\end{array}$

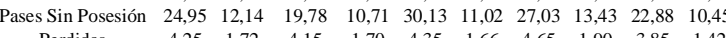
$\begin{array}{lllllllllll}\text { Perdidas } & 4,25 & 1,72 & 4,15 & 1,70 & 4,35 & 1,66 & 4,65 & 1,90 & 3,85 & 1,42\end{array}$ $\begin{array}{lllllllllll}\text { Recuperaciones } & 4,25 & 1,72 & 4,35 & 1,66 & 4,15 & 1,70 & 4,65 & 1,90 & 3,85 & 1,42\end{array}$ $\begin{array}{lrlllllllll}\text { Tiempo } & 99,40 & 29,46 & 99,40 & 29,61 & 99,40 & 29,61 & 104,25 & 30,06 & 94,55 & 28,39\end{array}$ $\begin{array}{lllllllllll}\text { Tiempo Posesiones } & 50,04 & 17,01 & 55,25 & 15,74 & 44,83 & 15,15 & 52,13 & 18,75 & 47,95 & 15,02\end{array}$

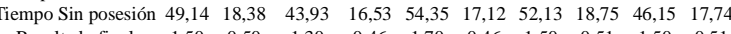
$\begin{array}{lllllllllll}\text { Resultado final } & 1,50 & 0,50 & 1,30 & 0,46 & 1,70 & 0,46 & 1,50 & 0,51 & 1,50 & 0,51\end{array}$ Leyenda: (NT) alude a la metodología basada en uso de las nuevas tecnologías (NT), mientras que (CO) hace referencia a la metodología tradicional basado en la comunicación oral y la enseñanza de la técnica.

Si se atiende a las comparaciones realizadas, reflejadas en la Tabla2, se aprecian diferencias significativas entre el pre-test y el post-test ( $p=$ $.000 p<.001)$, así como entre las dos diferentes intervenciones, CO o NT $(p=.000 p<.001)$ y el resultado, donde se aprecian diferencias entre ganadores y perdedores $(p=.008 p<.01)$. En relación al análisis previo y posterior a las intervenciones se aprecian diferencias en el número de posesiones, las pérdidas y las recuperaciones observadas ( $p$ $=.003 p<.01$ ). Analizando las diferentes variables consideradas, se aprecian diferencias significativas entre el grupo $\mathrm{CO}$ y NT en el número de pases con y sin posesión de balón y el tiempo de posesión y sin posesión de la pelota $(p=.000 p<.001)$, así como el resultado final ( $p$ $=.008 p<.01$ ). Si atendemos al resultado final se aprecian diferencias entre la duración de la jugada en posesión y sin posesión de balón ( $p=$ $.012 p<.05)$.

Al contemplar los análisis de diferencias de medias mediante la prueba U de Mann Whitney, presentados en la Tabla 2, y considerando los grupos de intervención (NT y CO) por separado, se aprecian diferencias significativas entre el número de pases con y sin posesión para ambos grupos $(p=.003 p<.01)$ para el grupo que siguió una metodología $\mathrm{CO}(p=.002 p<.01)$ y para el grupo cuya intervención siguió una metodología NT. En relación a la duración de las jugadas tanto en posesión de balón como sin ella, se aprecian diferencias significativas ( $p=$ $.000 p<.001$ ) para el grupo cuya intervención siguió una metodología $\mathrm{CO}(p=.001 p<.01)$ y para el grupo que siguió la intervención NT. En este sentido, en el grupo NT también se aprecian diferencias en función del resultado $(p=.013 p<.05)$. Así como también se halla significación estadística en relación al resultado final si se analiza la muestra considerando ganadores y perdedores $(p=.000 p<.01$ ) para ambos grupos (NT y CO).

Mientras que si se atiende a las comparaciones realizadas en categoría alevin, la Tabla 3 se aprecian diferencias significativas entre el pre-test y el pos-test $(p=.000 p<.001)$, así como entre las dos diferentes intervenciones, CO o NT ( $p=.000 p<.001)$ y el resultado, donde se
Tabla 2

Valores de las comparaciones entre grupos realizadas en función del test (pre-post), grupo (NT o CO) del inicio de la jugada o no y del

resultado (ganador o perdedor) en categoría benjon

\begin{tabular}{|c|c|c|c|c|c|c|c|c|c|c|c|c|}
\hline & Grupo & Test & Inicio & N. Pos & Pases & Pases SP & Per & Recup & $\mathrm{T}$ & T. Pos & T. SP & Resul \\
\hline Pre-Post & 1 & ,000 & 1 & ,003 (,32) & ,368 (,11) & ,368 (,11) & ,003 (,32) & , 003 (,32) & ,134 (,13) & , 213 (,10) & ,213 (,10) & $1(0)$ \\
\hline Grupo & ,000 & 1 & 1 & ,691 (,02) &, $000(, 43)$ & ,000 (-,43) & ,698 (-,02) & (698 & $1(0)$ & , 000 (,55) & ,000 (,55) & , 008 (-,29) \\
\hline Inicio & 1 & 1 & ,000 & , 362 & 1 & 1 & ,479 &, 479 & 1 & 139 &, 139 & 076 \\
\hline Resultado & ,008 & 1 & ,076 & ,362 & ,056 & ,056 & ,479 & , 479 & 1 & 012 & ,012 & ,000 \\
\hline \multicolumn{13}{|c|}{ Análisis Grupo CO } \\
\hline Pre-Post & - & - & 1 & ,028 (,29) & ,372 (-,34) & ,424 (,51) & ,031 (,34) & ,041(,29) & ,298 (,13) & ,025 (-,47) & ,914 (,62) & ,523(,29) \\
\hline Grupo & ,000 & - & 1 & 816 & 003 & 003 & 827 & 827 & 1 & 000 & ,000 & 217 \\
\hline Inicio & 1 & - & ,000 & 485 & 1 & 1 & ,565 & ,565 & 1 & ,386 & ,386 & 217 \\
\hline Resultado & ,217 & - & ,217 & 485 & ,228 & ,228 & ,565 & ,565 & 1 & ,130 & 130 & ,000 \\
\hline \multicolumn{13}{|c|}{ Análisis Grupo NT } \\
\hline Pre-Post & - & - & 1 & ,045 (,34) & ,424 (,51) & ,372 (-,34) & ,041 (,29) & , 031 (,34) & , 298 (,13) & ,914 (,62) & ,025 (-,47) & , 523 (-,29) \\
\hline Grupo & 000 & - & 1 & ,702 &, 002 &, 002 &, 764 &, 764 & 1 & ,001 & 001 & 013 \\
\hline Inicio & 1 & - &, 000 & ,461 & 1 & 1 &, 584 & ,584 & 1 & ,171 & 171 & ,217 \\
\hline Resultado & ,013 & - & ,217 & ,461 & 172 & ,172, & ,584 & ,584 & 1 & ,078 & ,078 & ,000 \\
\hline
\end{tabular}


aprecian diferencias entre ganadores y perdedores $(p=.000 p<.001)$. Analizando las diferentes variables consideradas se aprecian diferencias significativas entre el CO y NT en el número de pases con y sin posesión de balón y el resultado final $(p=.000 p<.001)$, asimismo se aprecian diferencias entre la duración de la jugada en posesión de balón $(p=.006 p<.01)$ y la duración de la jugada sin posesión $(p=.012 p<$ $.01)$. Si atendemos al resultado final se aprecian diferencias en el número de pases con y sin posesión de balón ( $p=.012 p<.05$ ).

Al considerar los análisis de diferencias de media mediante la prueba U de Mann Whitney considerando los grupos de intervención (NT y CO) por separado, se aprecian diferencias significativas entre el número de pases con y sin posesión para ambos grupos $(p=.003 p<.01)$ para el grupo que siguió una metodología CO y $(p=.005 p<.01)$ para el grupo cuya intervención siguió una metodología NT. En este sentido, en el grupo NT también se aprecian diferencias en función del resultado $(p=.017 p<.05)$ en relación al número de pases tanto en posesión de balón como en el equipo que no estaba en posesión del balón. Por otro lado, en el grupo que siguió una intervención CO se aprecian diferencias significativas en la comparación de medias realizadas entre el grupo (pre-post) en cuanto a la duración de las posesiones $(p=.001)$ para ambos grupos (con y sin posesión del balón).
En nuestro estudio no se aprecian diferencias significativas en relación al número de pérdidas y recuperaciones en ambos grupos, aunque sí se aprecian diferencias estadísticas al analizar el pre-test y post-test, lo que nos indica una mejora en el aprendizaje para el grupo NT frente al CO, debido al mayor número de recuperaciones y menores pérdidas realizadas por dicho grupo.

En este sentido, se puede considerar a la vista de los resultados obtenidos, y al contrario de los resultados expuestos por Carmona et al. (2015) en un estudio con jóvenes futbolistas, que el empleo de una estrategia en la práctica global que se relacionaría con nuestra propuesta de enseñanza usando las TIC, obtiene mejores resultados que el empleo de una metodología tradicional y analítica en cuanto al número de pases realizados (esto indica mejoras en el CO). Por otro lado, Sánchez, Yagüe, Fernández y Petisco (2014) observan una mejora en el desempeño de los deportistas en diferentes test analíticos sobre conducción y golpeo, al seguir una metodología de enseñanza analítica. No obstante, se les debe señalar, la baja representatividad de sus resultados, pues su evaluación del rendimiento se aleja de las acciones que se suceden en competición, ya que no usan como evaluación situaciones de juego real o semireal.
Tabla 3.

Valores de las comparaciones entre grupos realizadas en función del test (pre-pos), grupo (NT o CO), del inicio de la jugada o no, y del resultado (ganador o perdedor) en categoría alevin.

\begin{tabular}{|c|c|c|c|c|c|c|c|c|c|c|c|c|}
\hline & Grupo & Test & Inicio & N.Pos & Pases & Pases SP & Perd & Recup & $\mathrm{T}$ & T. Pos & T. SP & Res \\
\hline Pre-Pos & 1 & ,000 & 1 & ,053 (,23) & 209 (,17) & , 209 (,17) & ,056 (,23) & , 056 (,23) & , 065 (,16) & (310) & 139 (,16) & $1(0)$ \\
\hline Grupo & ,000 & 1 & 1 & ,456 (,06) & , 000 (,43) & , 000 (-,43) & ,530 $(-, 06)$ & , 530 (,06) & $1(0)$ & 006 (,32) & ,012 & , 000 (,40) \\
\hline Inicio & 1 & 1 & ,000 & 097 & 885 & 885 & 746 & 170 & 1 & 640 & 613 & 076 \\
\hline Resultado & ,000 & 1 & ,076 & ,097 & ,012 & ,012 & 158 & ,474 & 1 & ,419 & 418 & ,000 \\
\hline \multicolumn{13}{|c|}{ Análisis Grupo CO } \\
\hline Pre.-Pos & - & - & 1 & ,179 (,22) & ,180 (,21) & , 310 (16) & ,168 (,25) & ,199 (,20) & , 199 (,16) & ,004 (,42) & ,432 (-,13) & , 178 (,21) \\
\hline Grupo & ,000 & - & 1 & ,795 & 003 & 003 & ,794 & ,794 & 1 & ,001 & 001 & 217 \\
\hline Inicio & 1 & - & ,000 & 344 & 946 & 946 & 493 & 493 & 1 & ,507 & ,507 & 217 \\
\hline Resultado & 217 & - & ,217 & ,344 & 291 & 291 & ,493 & ,493 & 1 & ,417 & 417 & ,000 \\
\hline \multicolumn{13}{|c|}{ Análisis Grupo NT } \\
\hline Pre-Pos & - & - & 1 & , 177 (,24) & , 310 (,16) & , 180 (21) & , 199 (,20) & , 168 (,25) & (199 & , 151 & ,004 (,43) & , 178 (-,21) \\
\hline Grupo & ,000 & - & 1 & 443 & ,005 & ,005 & 549 & ,549 & 1 & 1 & 1 & ,000 \\
\hline Inicio & 1 & - & ,000 & 147 & ,797 & ,797 & 210 & ,210 & 1 & 1 & 1 & 217 \\
\hline Resultado & ,000 & - & ,217 & 147 & ,017 & 017, & 160, & ,749 & 1 & 1 & 1 & ,000, \\
\hline
\end{tabular}

\section{Conclusión}

A raíz de este trabajo, se comprueba que el uso de las TIC y el empleo del modelo de enseñanza comprensivo, mejora el aprendizaje de algunas acciones técnicas y su transferencia a la dinámica del juego en waterpolo. Puesto que se observan jugadas más largas, un mayor número de pases y recuperaciones, así como un menor número de pérdidas. Por lo que puede ser recomendable su uso en el proceso de enseñanza-aprendizaje en waterpolo, frente al planteamiento tradicional basado en la mecanización técnica de las acciones motrices en base a los resultados aquí expuestos.

\section{Discusión}

Este trabajo se ha desarrollado con el objetivo de analizar si el uso de dos metodologías de enseñanza-aprendizaje diferentes, una asociada al modelo comprensivo y el uso de las nuevas tecnologías y la otra propuesta basada en la comunicación oral y la enseñanza tradicional, influye en el aprendizaje de la dinámica del juego en waterpolo en categoría benjamín y alevín. Esto sirve como aproximación al estudio de la adquisición de las habilidades motrices y cognitivas complejas que se dan en varios deportes y que González, García, Vicedo y Contreras (2011), han estudiado en jugadores de fútbol. En el caso concreto del waterpolo no se conocen estudios previos, por lo que coincidimos con French y Thomas (1987) en que se necesita mayor investigación en el campo del conocimiento procedimental en este deporte.

Por otro lado, la investigación realizada por Durán y Lasierra(1994) y por Turner y Martinek (1992) en jugadores de hockey hierba consistente en comparar su versión estructural con otra centrada en la enseñanza de las habilidades técnicas, no llegó a conclusiones definitivas sobre la mayor bondad de una sobre la otra. En esta línea McPherson y French (1991) investigan sobre la eficacia de ambos modelos en la enseñanza del tenis y evalúan mediante test de conocimiento técnico y de rendimiento, donde encontraron que el grupo orientado a la técnica mejoró en los componentes cognitivos (test de conocimiento y decisiones durante el juego) sin instrucción directa o explícita. Sin embargo, el grupo orientado a la táctica no mejoró en la técnica hasta que se abordó ésta directamente (Méndez, 1999).

\section{Limitaciones}

Una de las limitaciones que presenta este estudio ha sido no considerar el momento de la temporada en que se realizó la intervención, lo cual puede comprometer los valores y posibles variables psicológicas como la ansiedad, la autoconfianza y la percepción de competencia de los jugadores y por tanto modificar los resultados aquí expuestos, así como la influencia del entorno social y del propio grupo de deportistas.

Tampoco se tuvo en consideración la existencia de portero y portería y su influencia en el aumento de la frecuencia cardíaca o la percepción de esfuerzo en cada grupo tal y como se ha indicado en estudios similares con futbolistas (Casamichana, Castellano \& Hernández-Mendo, 2014; Clemente, Wong, Martins \& Mendes, 2015; Falces, Casamichana, Sáez, Requena, Carling \& Suárez, 2015; Febré et al., 2015).

Por último, otra de las limitaciones del presente estudio es su baja reproductibilidad debido al escaso número muestral y la variación de normativa reglamentaria que rige el deporte en España en esta categoría, donde cada región posee una normativa diferente en cuanto al número de jugadores, duración del tiempo del encuentro y de la superioridad numérica o la existencia de esta, tamaño de la portería, peso y tamaño del balón y del campo de juego.

\section{Futuras líneas}

A raíz de este trabajo, se abren varias líneas de investigación. Una 
inicial, consiste en evaluar el componente motivacional (diversión) del deportista y la satisfacción subyacente a la intervención y programa realizado (García, Leo, Martín \& Sánchez, 2008; Castro, Zurita, Martínez, Chacón \& Espejo, 2016). Por otro lado, podría considerarse evaluar el feedback ofrecido por el entrenador y el clima de autonomía generado (Meng \& Keng, 2016) durante los test y la intervención. Así como el desempeño individual de cada jugador y la percepción de eficacia y aprendizaje del propio deportista (Gómez, Jiménez \& Sánchez, 2015) lo cual puede haber condicionado los resultados aquí expuestos.

En este sentido, además de las variables consideradas, futuros estudios deberían considerar realizarse con deportistas de mayor edad y diferente nivel deportivo (Sánchez, Courel \& Cañas, 2016), donde además se debería evaluar la ocupación del espacio, la distancia de los pases, los desplazamientos (relacionados con los objetivos de conservar, progresar o conseguir el objetivo) realizados por los deportistas (Serra \& García, 2017) y metros recorridos, tal y como hacen Carmona et al. (2015) y Silva, Esteves, Correia, Davids, Araújo y Garganta (2015) en futbolistas (mediante posicionamiento), así como su frecuencia cardíaca. Asimismo, debería considerarse contabilizar el rendimiento no sólo del jugador que lleva el móvil, tal y como se ha hecho en los pocos estudios de referencia, sino el de todos los participantes. Por lo que cobra relevancia analizar en función de las aportaciones de Bayer (1992) la toma de decisiones y ejecución de la habilidad y su adecuación al contexto (conservar, progresar y conseguir el objetivo) implicados en el marco ofensivo y que no son portadores del balón, o de las decisiones tomadas por los jugadores del equipo defensor sobre cuando moverse y hacia dónde o a quién cubrir (González et al., 2011).

\section{Referencias}

Abad, M. T. (2008). Propuesta de enseñanza del Tenis de Mesa en Educación Primaria y Secundaria. Retos: nuevas tendencias en educación física, deporte y recreación, 13(1), 33-38.

Abad, M. T., Giménez, F. J., Robles, J., \& Rodríguez, J. M. (2011). Perfil, experiencia y métodos de enseñanza de los entrenadores de jóvenes futbolistas en la provincia de Huelva. Retos: nuevas tendencias en educación física, deporte y recreación, 20, 21-25.

Abad, M. T., Benito, P. J., Giménez, F. J., \& Robles, J. (2013). Fundamentos pedagógicos de la enseñanza comprensiva del deporte: Una revisión de la literatura. Cultura, ciencia y deporte: revista de ciencias de la actividad física y del deporte de la Universidad Católica de San Antonio, 23, 137-146.

Anguera, M. T. (2003). Observational methods (general). En R. Fernández Ballesteros (Ed.). Encyclopedia of Psychological Assessment, 2, pp. 632-637. London: Sage.

Argudo, F. M., Alonso J. I., \& Fuentes, F. (2005). Computerized registration for tactical quantitative evaluation in water polo. Polo partido v1.0. Proceedings of the 5th International Symposium Computer Science in Sport, Croatia.

Bayer, C. (1986). La enseñanza de los juegos deportivos colectivos. Barcelona: Hispano europea.

Bayer, C. (1992). La enseñanza de los juegos deportivos colectivos. Barcelona: Hispano Europea.

Blomqvist, M., Luhtanen, P., \& Laakso, L. (2001). Comparison of two types of instruction in badminton. European Journal of Physical Education, 6(2), 139-155. (DOI: 10.1080/1740898010060206).

Bunker, D., \& Thorpe, R. (1982). A model for the teaching of games in secondary schools. Bulletin of Physical Education, 18(1), 1-4.

Cabero, J., \& Marín, V. (2014). Educational possibilities of social networks and group work. University students' perceptions. Comunicar, 42(XXI), 165-172. (DOI: http://dx.doi.org/10.3916/C422014-16).

Carmona, D., Luján, G., Francisco, J., \& Olmedilla, A. (2015). Efectos de un programa de formulación de objetivos y moldeamiento del pase en jóvenes jugadores de fútbol. Revista de psicología del deporte, 24(1), 81-88. (goo.gl/88A7j2).

Casamichana, D., Castellano, J., González, A., García, H., \& García, J.
(2011). Demanda fisiológica en juegos reducidos de fútbol con diferente orientación del espacio. RICYDE. Revista internacional de ciencias del deporte, 7(23), 141-154. (http://www.cafyd.com/ REVISTA/02306.pdf)

Casamichana, D., Castellano, J., \& Hernández-Mendo, A. (2014). La Teoría de la Generalizabilidad aplicada al estudio del perfil físico durante juegos reducidos con diferente orientación del espacio en fútbol. RICYDE. Revista internacional de ciencias del deporte, 37(10), 194-205. (http://dx.doi.org/10.5232/ricyde2014.03702).

Castejón, F. J. (2015). La investigación en iniciación deportiva válida para el profesorado de educación física en ejercicio. Retos: nuevas tendencias en educación física, deporte y recreación, 28(2), 263269.

Castejón, F. J., Martínez, L. F., del Campo, J., \& Argudo, F. M. (2011). Análisis de la documentación para la formación de entrenadores de base en baloncesto. Revista internacional de medicina y ciencias de la actividad física y el deporte, 11(42), 255-277. (http:// cdeporte.rediris.es/revista/revista42/artanalisis200.htm)

Castro, M., Zurita, F., Martínez, A., Chacón, R., \& Espejo, T. (2016). Clima motivacional de los adolescentes y su relación con el género, la práctica de actividad física, la modalidad deportiva, la práctica deportiva federada y la actividad física familiar. RICYDE. Revista internacional de ciencias del deporte, 45(12), 262-277). (http:// dx.doi.org/10.5232/ricyde2016.04504).

Clemente, F. M., Wong, D. P., Martins, F. M., \& Mendes, R. S. (2015). Differences in U14 football players' performance between different small-sided conditioned games. RICYDE. Revista internacional de ciencias del deporte, 42(11), 376-386. (http://dx.doi.org/10.5232/ ricyde2015.04206)

Cohen, J. (1988). Statistical power analysis for the behavioural sciences. Hillsdale, NJ: Erlbaum.

Colás, P., González, T., \& de Pablos, J. (2013). Young people and social networks: motivations and preferred uses. Comunicar, 40(XX), 15-23. (DOI: http://dx.doi.org/10.3916/C40-2013-02-01).

Dellal, A., Hill-Haas, S., Lago, C., \& Chamari, K. (2011). Small-sided games in soccer: amateur vs. professional players' physiological responses, physical, and technical activities. The Journal of Strength y Conditioning Research, 25(9), 2371-2381.

del Valle, S., Mendoza, N., Sánchez, M., \& de la Vega, R. (2007). Toma de conciencia de las situaciones y competencia deportiva. RICYDE. Revista internacional de ciencias del deporte, 9(3), 48-69.

Devís, J., \& Sánchez, R. (1996). La enseñanza alternativa de los juegos deportivos: antecedentes, modelos actuales de iniciación y reflexiones finales. Aprendizaje deportivo, 159-181.

Durán, C., \& Lasierra, G. (1994). Estudio experimental sobre didáctica aplicada a la iniciación de los deportes colectivos. Revista de investigación y documentación sobre las ciencias de la educación física y el deporte, 7, 92-128.

Falces, M., Casamichana D., Sáez, E., Requena, B., Carling, C., \& Suárez, L. J. (2015). The presence of the head coach during a smallsided game: effects on players' internal load and technical performance. RICYDE. Revista internacional de ciencias del deporte, 41(11), 245-257. (http://dx.doi.org/10.5232/ricyde2015.041).

Febré, R., Chirosa, L. J., Casamichana, D., Chirosa, I., Marín, I., \& Pablos, C. (2015). Influencia de la densidad de jugadores sobre la frecuencia cardíaca y respuestas técnicas en jóvenes jugadores de fútbol. RICYDE. Revista internacional de ciencias del deporte, 40(11), 116-128. (http://dx.doi.org/10.5232/ricyde2015.04002).

French, K. E., \& Thomas, J. R. (1987). The relation of Knowledge development to children's basketball performance. Journal of Sport Psychology, 9, 15-32.

García, P. (2009). Evaluación cuantitativa de la desigualdad numérica temporal simple con posesión mediante observación sistemática en waterpolo. Tesis doctoral sin publicar, Universidad Autónoma de Madrid, Madrid, España.

García, T., Leo, F. M., Martín, E., \& Sánchez, P. A. (2008). El compromiso deportivo y su relación con factores disposicionales y situacionales contextuales de la motivación. RICYDE. Revista internacional de ciencias del deporte, 12(4), 45-58. 
García-Valcárcel, A., Basilotta, V., \& López, C. (2014). ICT in collaborative learning in the classrooms of primary and secondary education. Comunicar, 42(XXI), 65-74. (DOI: http://dx.doi.org/ 10.3916/C42-2014-06).

Gómez, M., Roses, S., \& Farias, P. (2012). The academic use of social networks among university students. Comunicar, 38(XIX), 131138. (http://dx.doi.org/10.3916/C38-2012-03-04)

Gómez, A., Jiménez, F., \& Sánchez, C. R. (2015). Desarrollo de la Autonomía del Alumnado de Primaria en Educación Física a través de un proceso de investigación-acción. RICYDE. Revista internacional de ciencias del deporte, 42(11), 310-328. (http://dx.doi.org/ 10.5232/ricyde2015.04201).

González, S., García, L. M., Vicedo, J. C., \& Contreras, O. R. (2011). Conocimiento táctico y la toma de decisiones en jóvenes jugadores de fútbol (10 años). Revista de psicología del deporte, 20(1), 79-97.

González, S., Ibáñez, S. J., Feu, S., \& Galatti, L. R. (2017). Programas de intervención para la enseñanza deportiva en el contexto escolar, PETB y PEAB: Estudio preliminar. Retos. Nuevas tendencias en Educación Física, Deporte y Recreación, 31(2), 107-113.

Gordillo, A. (1992). Orientaciones psicológicas en la iniciación deportiva. Revista de psicología del deporte, 1(1), 27-36.

Gray, S., \& Sproule, J. (2011). Developing pupils’ performance in team invasion games. Physical Education \& Sport Pedagogy, 16(1), 1532.

Gréhaigne, J. F., Wallian, N., \& Godbout, P. (2005). Tactical-decision learning model and students' practices. Physical Education \& Sport Pedagogy, 10(3), 255-269. (https://doi.org/10.1080/ 17408980500340869).

Gubacs-Collins, K. (2007). Implementing a tactical approach through action research. Physical Education \& Sport Pedagogy, 12(2), 105 126. (https://doi.org/10.1080/17408980701281987).

Guillen, L., Copello, M., Gutierrez, M., \& Guerra, J. R. (2018). Metodología para el perfeccionamiento del proceso de enseñanza-aprendizaje de los elementos técnicos-tácticos en los deportes de combate. Retos. Nuevas tendencias en Educación Física, Deporte y Recreación (34), 33-39.

Guillen, L., Herrera, A. P., \& de la Rosa, Y. A. (2018). Las herramientas tecnológicas TIC' s como elemento alternativa para el desarrollo del componente físico. Retos. Nuevas tendencias en Educación Física, Deporte y Recreación (34), 222-229.

Holt, J. E., Ward, P., \& Wallhead, T. L. (2006). The transfer of learning from play practices to game play in young adult soccer players. Physical Education y Sport Pedagogy, 11(2), 101-118. (https:// doi.org/10.1080/17408980600708270).

Jiménez, F. (2011). Análisis estructural de las situaciones de enseñanza en los deportes colectivos. Acción motriz, 6, 39-57.

Katis, A., \& Kellis, E. (2009). Effects of small-sided games on physical conditioning and performance in young soccer players. Journal of Sports Science and Medicine, 8, 374-380.

León, O., \& Montero, I. (2003). Métodos de investigación en Psicología y Educación. Madrid: McGraw-Hill.

Light, R., \& Robert, J. E. (2010). The impact of Game Sense pedagogy on Australian rugby coaches' practice: a question of pedagogy. Physical Education \& Sport Pedagogy, 15(2), 103-115. (DOI: 10.1080/17408980902729388).

Light, R., \& Tan, S. (2006). Culture, embodied experience and teachers' development of TGfU in Australia and Singapore. European Physical Education Review, 12(1), 99-117. (DOI: 10.1177/ 1356336X06060659).

Little, T., \& Williams, A. G (2006). Suitability of soccer training drills for endurance training. The Journal of Strength \& Conditioning Research, 20(2), 316-319. (https://doi.org/10.1519/R-17094.1).

López, V., \& Castejón, F. J. (2005). La enseñanza integrada técnicotáctica de los deportes en edad escolar. Explicación y bases de un modelo. Apunts. Educación física y deportes, 1(79), 40-48.

Marín, V., Negre, F., \& Pérez, A. (2014). Construction of the foundations of the PLE and PLN for collaborative learning. Comunicar, 42(XXI), 35-43. (DOI: http://dx.doi.org/10.3916/C42-2014-03)

McPherson, S. L., \& French, K. E. (1991). Changes cognitive strategies and motor skill in tennis. Journal of Sport and Exercise Psychology, 13, 26-41. (doi: 10.1123/jsep.13.1.26).

Méndez, A. (1999). Modelos de enseñanza deportiva: análisis de dos décadas de investigación. Revista digital lecturas de educación física y deportes, 13. http://www.efdeportes.com/

Meng, H. Y. \& Keng, J. W. (2016). The effectiveness of an AutonomySupportive Teaching Structure in Physical Education. RICYDE. Revista internacional de ciencias del deporte, 43(12), 5-28. (http:/ /dx.doi.org/10.5232/ricyde2016.04301).

Meroño, L., Calderón, A., \& Hastie, P. A. (2016). Effect of Sport Education on the technical learning and motivational climate of junior high performance swimmers. RICYDE. Revista internacional de ciencias del deporte. 44(12), 182-198. (http://dx.doi.org/ 10.5232/ricyde2016.04407).

Montero, I., \& León, O. G. (2007). A guide for naming research studies in psychology. International Journal of Clinical and Health Psychology, 7(3), 847-862.

Navarro, V., \& Trigueros, C. (2008). Líneas de investigación del juego motor y modelos de estudio en el ámbito de la Educación Física española (1990-2007). Revista iberoamericana de educación, 46(4), 5.

Parlebàs, P. (1981). Contribution à un lexique commenté en science de la'action motrice. París: INSEP.

Piaget, J. (1982). El criterio moral en el niño. Barcelona: Fontanella. Edición de 1983.

Rampinini, E., Impellizzeri, F. M., Castagna, C., Abt, G., Chamari, K., Sassi,A., \& Marcora, S.M. (2007). Factors influencing physiological responses to small-sided soccer games. Journal of Sports Sciences, 25(6), 659-66.

Robles, J. (2006). Estrategia en la práctica global vs. analítica en la iniciación al judo. Lecturas: Educación física y deportes, 95, 32.

Rossi, T., Fry, J. M., McNeill, M., \& Tan, C. W. (2007). The games concept approach (GCA) as a mandated practice: Views of Singaporean teachers. Sport, Education and Society, 12(1), 93-111.

Sánchez, B. J., Courel, J., \& Cañas, J. (2016). Valoración de la precisión del golpeo en jugadores de pádel en función de su nivel de juego. RICYDE. Revista internacional de ciencias del deporte, 45(12), 323-333. (http://dx.doi.org/10.5232/ricyde2016.04507).

Sánchez, J., Yagüe, J. M., Fernández, R. C., \& Petisco, C. (2014). Efectos de un entrenamiento con juegos reducidos sobre la técnica y la condición física de jóvenes futbolistas. RICYDE. Revista internacional de ciencias del deporte, 37(10), 221-234. (http://dx.doi.org/ 10.5232/ricyde2014.03704).

Serra, J., \& García, J. (2017). La problemática táctica, clave en el diseño representativo de tareas desde el enfoque de la pedagogía no lineal aplicada al deporte. Retos: nuevas tendencias en educación física, deporte y recreación, 32(2), 278-280.

Sevil, J., Abós, A., Julián, J. A., Murillo, B., \& García, L. (2015). Género y motivación situacional en Educación Física: claves para el desarrollo de estrategias de intervención. RICYDE. Revista internacional de ciencias del deporte, 41(11), 281-296. (http://dx.doi.org/ 10.5232/ricyde2015.04106).

Silva, P., Esteves, P., Correia, V., Davids, K., Araújo, D., \& Garganta, J. (2015). Effects of manipulations of player numbers vs. field dimensions on inter-individual coordination during small-sided games in youth football. International Journal of Performance Analysis in Sport, 15(2), 641-659.

Tallir, I. B., Lenoir, M., Valcke, M., \& Musch, E. (2007). Do alternative instructional approaches result in different game performance learning outcomes? Authentic assessment in varying game conditions. International Journal of Sport Psychology, 38(3), 2332.

Tejedor, F. J., García-Valcárcel, A., \& Prada, S. (2009). Medida de actitudes del profesorado universitario hacia la integración de las TIC. Comunicar, 33 (XVII), 115-124. (DOI:10.3916/c33-200903-002).

Thorpe, R., Bunker, D., \& Almond, L. (1986). Rethinking games teaching. Loughborough: Department of Physical Education and Sports Science. 